\title{
Ameliorative impact of mycophenolate mofetil on diabetic kidney disease; a review on current knowledge
}

\author{
Hamid Nasri* \\ Department of Nephrology, Isfahan University of Medical Sciences, Isfahan, Iran
}

\author{
*Correspondence to \\ Prof. Hamid Nasri, \\ Email:
}

hamidnasri@med.mui.ac.ir

Received 4 February 2018 Accepted 19 March 2018 Published online 25 April 2018

Keywords: Diabetic nephropathy, Micro-inflammation, Mycophenolate mofetil, Diabetic kidney disease

\begin{abstract}
Diabetic kidney disease as a common problem of diabetes can be the main cause of renal insufficiency in severe and untreated cases. Various aspects play a role in the progress of diabetic nephropathy, including gradual accumulation of AGEs (advanced glycation end-products), advancement in the pathway of polyol, oxidative stress, high filtration in glomeruli and increase in TGF- $\beta$ (transforming growth factor- $\beta$ ) expression. Inflammatory process is known as a definite factor in the development of diabetic nephropathy. Increased molecules that cause inflammation comprise chemokines, cytokines, C-reactive protein (CRP), and adhesion molecules. Since pathogenesis of diabetic nephropathy basically is due to inflammatory procedure, therefore, the first therapeutic goal is to relieve inflammation. Numerous studies on animals have shown that anti-inflammatory effects of drugs are beneficial in treating nephropathy related to diabetes, but protective effects of these drugs have not been recognized in humans. Some substances involved in renal protective and anti-inflammatory in diabetic animals comprise; thiazolidinedione, statins, spironolactone, immunosuppressant, GLP-1 receptor agonist, angiotensin II receptor antagonist (ARB), cholecystokinin, and glucagon-like peptide-1. Mycophenolate mofetil (MMF) is a biologically inactive compound that belongs to a group of drugs that acts to suppress or restrain immune system. Therefore, mycophenolate, by weakening the immune system, prevents rejecting transplanted organ in the body. Here are some studies on the effect of MMF on inflammation as a significant factor in the development of diabetic problems.
\end{abstract}

Citation: Nasri H. Ameliorative impact of mycophenolate mofetil on diabetic kidney disease; a review on current knowledge. Immunopathol Persa. 2018;4(2):e19. DOI:10.15171/ ipp.2018.19.

\section{Introduction}

Diabetic nephropathy (diabetic kidney disease) is detected as a usual complication of diabetes that can be the principal cause of kidney failure in intense and untreated cases. Diabetic kidney disease is commonly demonstrated by gradually deteriorating of albuminuria, ensued by a decline in glomerular filtration rate (GFR), which over years or decades directed to end-stage kidney disease (ESKD) in many individuals with type 1 or type 2 diabetes. Morphologic lesions of diabetic kidney disease show a relationship of glomerular podocyte damage with lesions of glomeruli and the fibrosing process in the interstitial area. Some factors play a role in the expansion of diabetic kidney disease, including oxidative stress, high filtration in glomeruli (1), gradual accumulation of advanced glycation endproducts (AGEs), advancement in the

\begin{abstract}
Key point
Various experimental studies showed mycophenolate mofetil (MMF) could decrease the accumulation of leukocytes and macrophages in kidney tissues. These data afford the support for the potential therapeutic impact of MMF on hypertrophy and apoptosis of podocyte probably through inhabitation of genes expression. It is possible that MMF regarded as a beneficial drug for diabetic kidney disease patients in near future.
\end{abstract}

pathway of polyol, increase in transforming growth factor- $\beta$ (TGF- $\beta$ ) expression (2) and finally activation of cascade inflammatory factors.

There are various therapeutic options which may slow the progression of diabetic nephropathy, however, it is important to identify exactly mechanism of diabetic kidney disease and additional therapeutic

Copyright $(2018$ The Author(s); Published by Nickan Research Institute. This is an open-access article distributed under the terms of the Creative Commons Attribution License (http://creativecommons.org/licenses/by/4.0), which permits unrestricted use, distribution, and reproduction in any medium, provided the original work is properly cited. 
for prevention of end-stage renal disease.

Numerous factors such as; cytokines, chemokines, C-reactive protein (CRP), and adhesion molecules may aggravate the inflammatory processes. Inflammatory cells (mainly macrophages) play significant roles not only in classic inflammatory diseases but also in the pathogenesis of diabetic nephropathy (3). Furthermore, adhesion molecules play an important role in inflammatory process. These molecules expressed on the surface of leukocytes and endothelial cells, which can stimulate the adhesion of leukocytes to the endothelial cells of the vessels, and finally lead to their transfer from the lumen of the vessels to the site of inflammation (4). In this review we sought to present the possible beneficial impact of mycophenolate mofetil (MMF) on diabetic kidney disease.

\section{Materials and Methods}

For this review, we used a variety of sources including PubMed/Medline, EBSCO, EMBASE, Web of Science, and Scopus, and also directory of open access journals (DOAJ). The search was conducted by using combinations of the following key words and/or their equivalents; mycophenolate mofetil, diabetic nephropathy, diabetic kidney disease, end-stage kidney disease, podocyte damage, oxidative stress, advanced glycation endproducts, transforming growth factor- $\beta$, Intercellular adhesion molecule-1, vascular adhesion molecule-1, endothelial cells, renin-angiotensin system, monocytes, tubulointerstitial area, interstitial area, mesangial cells, tubular epithelial cells, mycophenolic acid, guanine nucleotide synthesis pathway, apoptosis, podocytes and glomerulosclerosis.

\section{Mechanism of adhesion molecules}

Intercellular adhesion molecule-1 (ICAM-1), an adhesion molecule of the immunoglobulin superfamily that binds to b2 integrins of lymphocytes or macrophages, and vascular adhesion molecule-1 (VCAM-1) that involve in the attachment of leukocytes to the vascular wall and penetration into the wall of the vessels. Afterward, leukocytes can create proteolytic enzymes that cause tissue and organ injury. It is possible that leukocytes differentiate into foam cells that lead to the tissue inflammation processes. This is showed high expression of ICAM-1 occurs in response to several kinds of stimulation such as; pro-inflammatory cytokines, oxidative stress, protein kinase $\mathrm{C}$ activation and AGEs (5). A recent investigation presented, mice deficient of ICAM-1 can be resistant to nephropathy in experimental diabetic models, while treatment with anti-ICAM-1 monoclonal antibody prevents mononuclear cell infiltration into diabetic lesions in the glomeruli. Likewise, it is demonstrated that anti-ICAM-1 agents can prevent albumin secretion in the urine and also kidney damages due to nephropathy. Moreover, some studies showed that increasing the levels of ICAM-1 in plasma is related to elevated urinary albumin secretion and intensity of albuminuria in diabetic patients. Consequently, ICAM-1 may be activated by some factors such as; pro-inflammatory cytokines, shear stress, oxidative stress, protein kinase C, and AGEs (6).

On the other hand, the levels of VCAM-1, another adhesion molecule that elevates on the surface of on endothelial cells in diabetic animals, is associated with the severity of albuminuria in diabetic patients. Furthermore, VCAM-1 gene expression is increased in kidney biopsies from patients with diabetic kidney disease. Recent findings showed a correlation between levels of VCAM-1 and proportion of infiltrating immune cells. In addition, increased level of VCAM-1 is related to the urine secretion of albumin in patients with type 2 diabetes and mortality rate of diabetic kidney disease too (7).

\section{Molecular mechanism of diabetic kidney disease} When leukocytes migrate into kidney tissue (macrophages) could elaborate pro-inflammatory and pro-fibrotic cytokines therefore, produce structural impairment of the tissue and stimulation of renin-angiotensin system (RAS) followed by a decline in GFR. The role of chemokines in the pathogenesis of diabetic nephropathy is stimulating the extension of inflammatory cells into inflammatory sites by binding to their receptors (8).

There are several kinds of chemokines, such as; monocyte chemoattractant protein-1 or C-C motif chemokine 2 (CCL2), C-X3-C motif chemokine 1 (CX3CL1) and C-C motif chemokine 5 (CCL5,) that play important roles in the pathogenesis of diabetic kidney disease. It is demonstrated that CCL2 can induce migration more monocytes into the kidney. Furthermore, CCL2 gene expression increased in the tubulointerstitial area of patients with diabetic kidney disease who harbored a renal biopsy. This disorder may be connected to protein kinase $\mathrm{C}$, oxidative stress, high glucose concentration, AGEs and elevation of angiotensin II (9).

CX3CL1, fractalkine, endorses the migration of mononuclear cells and adhesion between cells consequently, they can enhance infiltration of monocytes into the interstitial area and make an interstitial injury $(8,9)$.

CCL5, RANTES, is expressed in mesangial cells and tubular epithelial cells of diabetic kidney disease and CCL5 expression is increased by angiotensin II and proinflammatory cytokine $(8,9)$.

\section{Therapeutic agents for diabetic nephropathy}

Since one of the main causes diabetic kidney disease is increasing of inflammatory factors, therefore, the fundamentally goal therapeutic is the improvement of inflammatory process. Various, in vitro and in vivo investigations on animals have shown that antiinflammatory effects of drugs are beneficial in treating nephropathy related to diabetes, but protective impacts of these drugs have not been determined in humans. Some of the agents regarded as the protective and antiinflammatory substances in diabetic animals comprise; angiotensin converting enzyme inhibitors (ACEI), statins, 
thiazolidinedione, angiotensin II receptor antagonist (ARB), spironolactone, immunosuppressant, GLP-1 receptor agonist, cholecystokinin, and glucagon-like peptide-1. Angiotensin II receptor antagonist/angiotensin converting enzyme inhibitors can inhibit pathways dependent on nuclear factor-kB (NF-kB), hence exerts the protective impacts on kidneys (10). Anti-inflammatory impact of statin is related to blockage of small-G proteins and inflammatory pathways dependent on NF-kB. Despite current treatment such as; glycemic control, blood pressure control, with emphasis on agents targeting the reninangiotensin system. These strategies still present defective protection against progression of kidney damage in diabetic kidney disease. Hence, newer therapeutic agents are necessary for amelioration of current treatments that have potential to affect main mechanisms contributing to the pathogenesis of diabetic kidney disease (11).

MMF, a therapeutic agent that administered to prevent allograft rejection, to suppress leukocytes or macrophages penetration to the tissues and proliferation of inflammatory cells. MMF, also, suppresses the expression of ICAM-1 and VCAM-1in the experimental models of diabetic kidney disease. Moreover, MMF regarded as an anti-proliferative and immunosuppressive agent on the progression of glomerular injury in diabetic rats (12).

\section{Structure of MMF and its mechanism}

MMF is a biologically inactive compound that can be metabolized in the body to mycophenolic acid. This agent belongs to a group of drugs that acts to suppress or restrain immune system. Actually, by weakening the immune system, mycophenolate, prevents rejecting transplanted organs. According to previous studies, there are various mechanisms that MMF can contribute to restraining immune system, one of them is inhibiting the enzyme required to grow $\mathrm{T}$ cells and $\mathrm{B}$ cells (12). Moreover, MMF is one of the 2-ethyl ester derivatives of mycophenolic acid that can non-competitively and reversibly inhibit inosine monophosphate dehydrogenase, thus, prevent the guanine nucleotide synthesis pathway to additional block the synthesis of DNA and RNA (13). This issue has been revealed, the major pharmacological effects of MMF including, inhibition of leukocytes and macrophages cell proliferation, reduction of antibody production, and inhibition of cell surface adhesion molecules. In recent years, MMF administered as an antirejection treatment after organ transplantation. However, some diabetic animal studies showed treatment by MMF had not any significant changes in blood pressure and blood glucose although, levels of urinary albumin excretion significantly decreased. Furthermore, MMF can significantly decrease monocyte/macrophage infiltration in renal tissues therefore, the renal tissue is effectively protected against the progression of damage tissue (14). Some studies show monocyte chemoattractant protein-1 (MCP-1) gene expression effectively inhibited by MMF. Generally, MCP-1 is a cytokine that has a monocyte- specific chemotactic function and involves in monocyte/ macrophage infiltration, therefore it plays an important role in the pathogenesis of diabetic kidney disease.

On the other hand, glomerular hypertrophy is a trademark of diabetic kidney disease that causes extracellular matrix (ECM) expansion followed by structural and functional of renal tissue change. Finally, hypertrophy or apoptosis occurs in podocytes. Therefore, podocyte injury has detected to play an essential role in diabetic kidney disease. Reduction or differentiation of podocyte is main mechanisms driving glomerulosclerosis and progressive deterioration of kidney function $(14,15)$.

Some studies supported that MMF could significantly inhibit hypertrophy and apoptosis of podocyte. Additionally, researchers elucidated that MMF can be reduced the cell cycle arrest and apoptosis of podocyte with the underling mechanism that MMF causes a significant increase of p27 and p21 expression. Therefore, it is possible that MMF can inhibit podocyte hypertrophy by significantly up-regulated p27 and p21 expression. Likewise, MMF is able to inhibit podocyte apoptosis through a significantly increasing cleaved of caspase- 3 and bax expression and decreasing of $b c l-2$ gene expression. Finally, MMF can contribute to inhibit cell cycle arrest and apoptosis of podocytes (16).

On the other hand, it is detected that MMF can decrease renal levels of TNF- $\alpha$ and IL17A in diabetic mice. Accordingly, it can reduce levels of IL-4 and IL-6 in kidney and plasma of diabetic mice (16).

\section{Conclusion}

In conclusion, recent experimental studies showed MMF could decrease the accumulation of leukocytes and macrophages in kidney tissues. These data afford the support for the potential therapeutic impact of MMF on hypertrophy and apoptosis of podocyte probably through inhabitation of genes expression. It is possible that MMF regarded as ad a beneficial drug for diabetic nephropathy patients in near future.

Author's contribution

$\mathrm{HN}$ is the single author of the paper.

Conflicts of interest

The author declared no competing interests.

Ethical considerations

Ethical issues (including plagiarism, data fabrication, double publication) have been completely observed by the author.

Funding/Support

None.

References

1. Magee GM, Bilous RW, Cardwell CR, Hunter SJ, Kee F, Fogarty DG. Is hyperfiltration associated with the future risk of developing diabetic nephropathy? A meta-analysis. Diabetologia. 2009;52:691-7. doi: 10.1007/s00125-0091268-0.

2. Ziyadeh FN, Sharma K, Ericksen M, Wolf G. Stimulation of 
collagen gene expression and protein synthesis in murine mesangial cells by high glucose is mediated by autocrine activation of transforming growth factor-beta. J Clin Invest. 1994;93:536-42. doi: 10.1172/JCl117004.

3. Ross R. Atherosclerosis-an inflammatory disease. N Engl J Med. 1999;14:115-26. doi: 10.1056/NEJM199901143400207.

4. Staunton DE, Marlin SD, Stratowa C, Dustin ML, Springer TA. Primary structure of ICAM-1 demonstrates interaction between members of the immunoglobulin and integrin supergene families. Cell. 1988;52:925-33.

5. Shikata K, Makino H. Microinflammation in the pathogenesis of diabetic nephropathy. J Diabetes Investig. 2013;4:142-9. doi: 10.1111/jdi.12050.

6. Lin J, Glynn RJ, Rifai N, Manson JE, Ridker PM, Nathan DM, et al. Inflammation and progressive nephropathy in type 1 diabetes in the Diabetes Control and Complications Trial. Diabetes Care. 2008;31:2338-43. doi: 10.2337/dc08-0277.

7. Rubio-Guerra AF, Vargas-Robles H, Lozano Nuevo JJ, Escalante-Acosta BA. Correlation between circulating adhesion molecule levels and albuminuria in type 2 diabetic hypertensive patients. Kidney Blood Press Res. 2009;32:1069. doi: 10.1159/000210554

8. Ruster C, Wolf G. The role of chemokines and chemokine receptors in diabetic nephropathy. Front Biosci. 2008;13:94455.

9. Chow FY, Nikolic-Paterson DJ, Ozols E, Atkins RC, Rollin BJ, Tesch $\mathrm{GH}$. Monocyte chemoattractant protein 1 promotes the development of diabetic renal injury in streptozotocin-treated mice. Kidney Int. 2006;69:73-80. doi: 10.1038/sj.ki.5000014.
10. Dai SM, Matsuno H, Nakamura H, Nishioka K, Yudoh K. Interleukin 18 enhances monocyte tumor necrosis factor a and interleukin $1 \mathrm{~b}$ production induced by direct contact with T lymphocytes: implications in rheumatoid arthritis. Arthritis Rheum. 2004;50:432-43. doi: 10.1002/art.20064.

11. Usui H, Shikata K, Matsuda M, Okada S, Ogawa D, Yamashita $\mathrm{T}$, et al. HMG-CoA reductase inhibitor ameliorates diabetic nephropathy by its pleiotropic effects in rats. Nephrol Dial Transplant. 2003;18:265-72.

12. Kitchin JE, Pomeranz MK, Pak G, Washenik K, Shupack JL. Rediscovering mycophenolic acid: A review of its mechanism, side effects, and potential uses. J Am Acad Dermatol. 1997;37:445-9.

13. Allison AC, Eugui EM. Mycophenolate mofetil and its mechanisms of action. Immunopharmacology. 2000;47:85118.

14. Utimura R, Fujihara CK, Mattar AL, Malheiros DM, Noronha IL, Zatz R. Mycophenolate mofetil prevents the development of glomerular injury in experimental diabetes. Kidney Int. 2003;63:209-16. doi : 10.1046/j.1523-1755.2003.00736.x.

15. Lv W, Zhang Y, Guan G, Li P, Wang J, Qi D. Mycophenolate mofetil and valsartan inhibit podocyte apoptosis in streptozotocin-induced diabetic rats. Pharmacology. 2013:92:227-34. doi: 10.1159/000354600.

16. Seo JW, Kim YG, Lee SH, Lee A, Kim DJ, Jeong KH, el al. Mycophenolate mofetil ameliorates diabetic nephropathy in db/db mice. Biomed Res Int. 2015;2015:301627. doi: $10.1155 / 2015 / 301627$. 